\title{
Role of echocardiography in screening for portopulmonary hypertension in liver transplant candidates: A meta-analysis
}

\author{
Xin Yin ${ }^{1,2}$, Yueming Shao ${ }^{1}$, Yu Zhang ${ }^{1}$, Hui Gao ${ }^{3}$, Tingting Qin ${ }^{1}$, Xiaoyu Wen ${ }^{\text {Corresp., } 1}{ }^{1}$, Chen Yang ${ }^{\text {Corresp. } 4}$ \\ ${ }^{1}$ Department of Hepatology, The First Hospital of Jilin University, Changchun, Jilin Province, China \\ 2 Chengdu Women's and Children's Central Hospital, Chengdu, Sichuan Province, China \\ 3 Department of Digestive System, The Hospital of Tai'an Municipal, Tai'an, Shandong Province, China \\ 4 Department of Bone and Joint Surgery, The First Hospital of Jilin University, Changchun, Jilin Province, China \\ Corresponding Authors: Xiaoyu Wen, Chen Yang \\ Email address: 15804301609@163.com, 15043031980@163.com
}

Objectives. To demonstrate the screening value of echocardiography for portopulmonary hypertension (POPH) in liver transplant candidates.

Design. Systematic review and meta-analysis

Background. POPH is a complication of end-stage liver disease that adversely affects the outcome of orthotopic liver transplant. There are no specific symptoms in the early stage of POPH. POPH reduce the survival rate of patients with end-stage liver disease specially if they are not diagnosed. Therefore, early detection may improve prognosis. The objective of this study is to explore the screening value of echocardiography on liver transplant candidates for screening of $\mathrm{POPH}$ compared to right heart catheterization (RHC).

Method. PubMed, EMBASE and the Cochrane Library were searched by two independent reviewers for potentially eligible studies published up to 30 June 2019 to retrieve data based on per-patient analysis. STATA, Meta-DiSc, and RevMan were applied to perform this meta-analysis.

Results. Our search yielded 1576 studies, of which 11 satisfied the inclusion criteria. The pooled sensitivity, specificity, positive likelihood ratio (PLR), negative likelihood ratio (NLR) and area under the summary receiver operating characteristic (SROC) curve (AUC) of echocardiography for POPH were 0.85 (95\% Cl, 0.65-0.94), $0.83(95 \% \mathrm{Cl}, 0.73-0.90), 4.99(95 \% \mathrm{Cl}, 3.03-8.21), 0.19(95 \% \mathrm{Cl}, 0.07-0.46)$, and 0.91 (95\% Cl, 0.88-0.93), respectively. Deeks' funnel plot did not indicate the existence of publication bias $(P=0.66)$.

Conclusions. Echocardiography, a noninvasive modality, provides superior screening for $\mathrm{POPH}$, but the diagnosis of POPH still requires $\mathrm{RHC}$.

\section{PROSPERO registration number}

CRD42019144589 
1 Role of echocardiography in screening for portopulmonary hypertension in liver transplant

2 candidates: A meta-analysis

3 Xin Yin ${ }^{1,2}$, Yueming Shao ${ }^{1}$, Yu Zhang ${ }^{1}$, Hui Gao ${ }^{3}$, Tingting Qin ${ }^{1}$, Xiaoyu Wen ${ }^{1}$, Chen Yang ${ }^{4}$

4 'Department of Hepatology, The First Hospital of Jilin University, Changchun, Jilin Province,

5 China

$6 \quad{ }^{2}$ Chengdu Women's and Children's Central Hospital, Chengdu, Sichuan Province, China

$7 \quad{ }^{3}$ Department of Digestive System, The Hospital of Tai'an Municipal, Tai'an, Shandong

8 Province, China

$9{ }^{4}$ Department of Bone and Joint Surgery, The First Hospital of Jilin University, Changchun, Jilin

10 Province, China

11 Corresponding author: Xiaoyu Wen ${ }^{1}$, Chen Yang ${ }^{4}$

12 Xinmin Street, Changchun, Jilin Province, 130021, China

13 Email address: 15804301609@163.com,15043031980@163.com 


\section{Abstract}

Objectives. To demonstrate the screening value of echocardiography for portopulmonary hypertension $(\mathrm{POPH})$ in liver transplant candidates.

Design. Systematic review and meta-analysis

Background. POPH is a complication of end-stage liver disease that adversely affects the outcome of orthotopic liver transplant. There are no specific symptoms in the early stage of POPH. POPH reduce the survival rate of patients with end-stage liver disease specially if they are not diagnosed. Therefore, early detection may improve prognosis. The objective of this study is to explore the screening value of echocardiography on liver transplant candidates for screening of POPH compared to right heart catheterization (RHC).

Method. PubMed, EMBASE and the Cochrane Library were searched by two independent reviewers for potentially eligible studies published up to 30 June 2019 to retrieve data based on per-patient analysis. STATA, Meta-DiSc, and RevMan were applied to perform this metaanalysis.

Results. Our search yielded 1576 studies, of which 11 satisfied the inclusion criteria. The pooled sensitivity, specificity, positive likelihood ratio (PLR), negative likelihood ratio (NLR) and area under the summary receiver operating characteristic (SROC) curve (AUC) of echocardiography for POPH were 0.85 (95\% CI, 0.65-0.94), 0.83 (95\% CI, 0.73-0.90), 4.99 (95\% CI, 3.03-8.21), 0.19 (95\% CI, 0.07-0.46), and 0.91 (95\% CI, 0.88-0.93), respectively. Deeks' funnel plot did not indicate the existence of publication bias $(P=0.66)$.

Conclusions. Echocardiography, a noninvasive modality, provides superior screening for POPH, 
43 but the diagnosis of POPH still requires RHC.

44 PROSPERO registration number

45

46

47

48

49

CRD42019144589

\section{Strengths and limitations of this study}

This is the first meta-analysis to assess the value of echocardiography as a screening tool for POPH.

The methodological quality of each study was assessed using Quality Assessment of Diagnostic Accuracy Studies 2 (QUADAS-2).

The limitation of this meta-analysis is that further related subgroup analysis was not conducted, although heterogeneity was high.

\section{Introduction}

Portopulmonary hypertension (POPH) is a type of pulmonary hypertension (PH) associated with portal hypertension, which is a rare complication of end-stage liver disease. In patients with portal hypertension, the occurrence of $\mathrm{PH}$ is reported to be $2 \%$ to $6 \%{ }^{1}$. The prevalence of POPH in patients who are candidates for liver transplant varies between $3 \%$ and $10 \%{ }^{2,3}$. A robust diagnosis of POPH requires the presence of portal hypertension and hemodynamic instability upon invasive right heart catheterization (RHC), namely, a mean pulmonary artery pressure $(\mathrm{mPAP}) \geq 25 \mathrm{mmHg}$, pulmonary vascular resistance $(\mathrm{PVR})>240$ dynes.s.cm ${ }^{-5}$ and pulmonary capillary wedge pressure $(\mathrm{PCWP})<15 \mathrm{mmHg}^{4}$. Female sex and autoimmune hepatitis are associated with an increased risk of $\mathrm{POPH}$, whereas hepatitis $\mathrm{C}$ infection is a protective factor against $\mathrm{POPH}^{5}$. 
64 The mean survival of POPH patients is approximately 15 months without medical intervention ${ }^{6}$.

65 To improve survival, patients with POPH should be treated with medication or undergo liver

66 transplantation. However, a multicenter study reported that the mortality rate of patients with

$67 \mathrm{POPH}$ is as high as $36 \%$ after liver transplantation ${ }^{7}$. Because the prevalence of $\mathrm{POPH}$ is

68

69 relatively low, it is not feasible for all patients to undergo invasive RHC. Furthermore, $60 \%$ of the patients have no obvious clinical symptoms when POPH is diagnosed ${ }^{8}$. Accordingly, there is a need for noninvasive methods to screen patients for POPH as early as possible. Estimated pulmonary artery systolic pressure (ePASP) on echocardiography is determined using the modified Bernoulli equation: ePASP $(\mathrm{mmHg})=4 \times \mathrm{TRV}^{2}+$ estimated right atrial pressure, with TRV representing the tricuspid regurgitant peak velocity ${ }^{9}$. The risk of $\mathrm{PH}$ is increases when indicated by echocardiography that the pulmonary artery is widened and right heart morphology is altered ${ }^{10}$. However, RHC should be performed to confirm the existence of POPH when the ePASP of a liver transplantation candidate is more than $50 \mathrm{mmHg}^{4}$. The existence of POPH can be excluded when the ePASP, as measured by echocardiography, is less than $30 \mathrm{mmHg}^{11}$. There have been many studies conducted on echocardiography as a screening tool for POPH, but each study reported different conclusions.

Therefore, we performed this meta-analysis, which may complement existing studies, to evaluate the accuracy of echocardiography compared to RHC as a screening method in liver transplantation patients.

\section{Materials and methods}

\section{Search strategy}


85 This meta-analysis was registered in PROSPERO, and the registration number was

86

87

CRD42019144589. PubMed, EMBASE and the Cochrane Library were searched by two independent reviewers (Xin Yin and Yueming Shao) for potentially eligible studies published up to 30 June 2019. The search terms were a combination of medical subject headings (MESH) and keywords. The search strategy was as follows: ("Portopulmonary hypertension" or "porto pulmonary hypertension" or "POPH" or "PPH" or "PPHTN") and ("echocardiography"). The "All fields" category was used for search. The retrieval strategies were adjusted according to the different databases and were confirmed after many pre-retrievals of the combination of words above. In addition, to check for potential studies, we scanned the references list of existing systematic reviews and meta-analyses relevant to our study.

\section{Inclusion and exclusion criteria}

All retrieved articles were screened by two independent reviewers (Xin Yin and Yueming Shao) according to the inclusion and exclusion criteria, and disagreement was resolved by a third author (Yu Zhang). The studies were required to meet the following criteria: (1) patients included in the studies were liver transplantation candidates who underwent echocardiography and RHC before liver transplantation; (2) the results of RHC served as a reference standard for diagnosis and severity; (3) a certain cut-off values for echocardiography was adopted to screen POPH patients; (4) effective tricuspid regurgitation was demonstrated by echocardiography and pulmonary artery pressure was estimated; and (5) the extracted data were available to calculate true positive, false positive, false negative and true positive values. The exclusion criteria of this study were as follows: (1) non-English articles; (2) case reports, conference abstracts, reviews, 
106

107

108

109

110

111

112

113

114

115

116

117

118

119

120

121

122

123

124

125

126

editorial materials, letters, and comments; and (3) studies involving the individuals.

\section{Data extraction and quality assessment}

Two independent reviewers (Xin Yin and Hui Gao) extracted the following information: first author, year, sample size, mean/median age, the number of POPH/non-POPH patients, cut-off value, false negative, false positive, true negative, true positive. Disagreement was solved by discussion and if necessary, a third reviewer (Tingting Qin) was involved to reach a consensus. Quality assessment was assessed by two independent researchers (Xiaoyu Wen and Chen Yang) using Quality Assessment of Diagnostic Accuracy Studies 2 (QUADAS-2).

\section{Statistical analysis}

The threshold effect was considered first. The $P$-value of the Spearman correlation coefficient was used to measure the threshold effect. A $P$-value greater than 0.05 indicated that there was no threshold effect and that further exploration into whether heterogeneity was caused by a nonthreshold effect was needed. The heterogeneity was evaluated by the value of the $I$-square statistic using the "midas" command based on a bivariate model of a hierarchical receiver operating characteristic (HSROC). The combined sensitivity, specificity, positive likelihood ratio (PLR), negative likelihood ratio (NLR), and their 95\% confidence intervals (CIs) were calculated and graphically shown using forest plots. A summary receiver characteristic curve (SROC) was applied to assess the screening accuracy of echocardiography, and the AUC was computed; the higher the AUC, the higher the screening value was. Deeks' funnel plot asymmetry test was applied to assess publication bias. This meta-analysis was conducted by STATA software (version 15.0, StataCrop, College Station, Texas, USA) and Meta-DiSc 1.4 ${ }^{12}$. Quality assessment 
127

128

129

130

131

132

133

134

135

136

137

138

139

140

141

142

143

144

145

146

147

was performed using Review Manager 5.3. $P<0.05$ was considered to be statistically significant.

Additionally, we combined the Pearson's correlation coefficient of echocardiography and RHC

in liver transplantation individuals using the "metacor" package of $\mathrm{R}$ software (version 3.5.3).

\section{Results}

\section{Literature search}

A total of 1089 articles were retrieved by electronic search after duplicates were excluded. Based on the inclusion and exclusion criteria, 11 full-text articles ${ }^{3,11,13-21}$ were ultimately included in the meta-analysis. A PRISMA flow diagram of the retrieved studies is shown in Figure 1.

\section{Basic characteristics and quality assessment}

In conclusion, 11 studies published between 2000 and 2019 involving 1160 liver transplant candidates were used in the analysis; 5 of the studies were from Europe, 4 were from the USA, and 2 were from Asia. Eight of the included studies were prospective design, and the remaining 3 studies were retrospective studies. The cut-off value of echocardiography in these studies varied from $30 \mathrm{mmHg}$ to $50 \mathrm{mmHg}$. Table 1 presents the detailed information of the included studies.

The results of the quality assessment suggested that the risk of bias was low and that the quality of the included studies was high (Supplementary File Figure S1).

\section{Meta-analysis}

The 11 eligible studies were pooled for the present meta-analysis of diagnostic tests. In the threshold analysis, the Spearman correlation coefficient was 0.210 , and the $P$-value was 0.536 , indicating that there was no threshold effect. Figure 2 shows the forest plots of sensitivity, which ranged from 0.55 to 1.00 (pooled, $0.85 ; 95 \% \mathrm{CI}, 0.65-0.94$ ), and specificity, which ranged from 

0.48 to 0.98 (pooled, $0.83 ; 95 \%$ CI, 0.73-0.90). The combined PLR was 4.99, and the combined NLR was 0.19, and these data are presented in the supplementary file (Figure S2). Figure S3 shows the screening odds ratio, which ranged from 5.24 to 415.80 (pooled, $26.90 ; 95 \%$ CI, 8.37- 
169

170

171

indicating that there was no significant publication bias.

\section{Discussion}

The purpose of echocardiography screening before liver transplantation is to identify patients with clinically significant POPH before surgery and improve their prognosis. Because of the invasiveness of this procedure and coagulation disorders in patients with end-stage liver disease, RHC cannot be used as a screening tool for liver disease patients. The guidelines of the American Association for the Study of Liver Disease (AASLD) suggest that all patients who are waiting for liver transplantation should be screened by echocardiography ${ }^{25}$. However, there have been no reports regarding the accuracy of echocardiography as a screening tool for POPH.

Our study confirms that the screening accuracy of echocardiography for POPH is clinically acceptable, showing a sensitivity of 0.85 (95\% CI, 0.65-0.94), specificity of 0.83 (95\% CI, 0.73 0.90), and area under the SROC curve of 0.91 (95\% CI, 0.88-0.93). Two meta-analyses were previously conducted to evaluate the diagnostic value of echocardiography in pulmonary hypertension (PH). A meta-analysis by de Surinder showed that the estimated sensitivities and specificity of echocardiography for patients with $\mathrm{PH}$ were $83 \%$ and $72 \%^{26}$, respectively.

Mohammed et $\mathrm{al}^{23}$ conducted a meta-analysis of 9 studies among patients with $\mathrm{PH}$ and found that echocardiography had a sensitivity of $88 \%$ and a specificity of $56 \%$ for $\mathrm{PH}$ patients. However, these two studies were meta-analyses based on echocardiography as a diagnostic tool for patients with PH. Our article looked at a special group of patients with $\mathrm{PH}$ and is a comprehensive study of reports up to 31 June 2019. Our results showed that echocardiography had high sensitivity and specificity for detecting POPH in liver transplantation candidates. 
190

191

Approximately 9.5\%-22\% of liver transplant candidates have no detectable tricuspid

regurgitation (TR) by Doppler echocardiography ${ }^{3,24}$. However, TR is nearly invariable in patients with $\mathrm{PH}^{27}$. Moreover, the specificity of echocardiography as a screening tool for POPH was 0.83 according to 11 studies. Therefore, the risk of missing POPH because of the absence of TR might be extremely low ${ }^{15}$. Although our results further confirmed the screening accuracy of echocardiography in POPH patients, echocardiography does not differentiate between precapillary and postcapillary $\mathrm{PH}^{28}$. Therefore, $\mathrm{RHC}$ is necessary to characterize the specific hemodynamic patterns. Importantly, these patterns may require different therapeutic approaches based on volume status. In addition, the latest guidelines ${ }^{29}$ indicate that an mPAP of $20 \mathrm{mmHg}$ should be considered the upper limit of normal. Hence, the sensitivity of echocardiography diagnosis for POPH may be underestimated by existing studies.

There were several implicit limitations in our meta-analysis. First, we included only studies published in PubMed, EMBASE and the Cochrane Library, and we excluded abstracts, letters to the editor and articles written in languages other than English. This may have led to publication bias. Second, the time interval between echocardiography and RHC was different. The longer the period between echocardiography and RHC, the higher the chance that the hemodynamic status of patients will change. Third, 1 of the 11 articles estimated right atrial pressure based on a fixed value of $10 \mathrm{mmHg}^{18}$. In other studies, right atrial pressure was estimated using the inferior vena cava diameter. The use of the jugular venous pressure for clinical estimates does not allow reliable measure of right atrial pressure and is less satisfactory than using a fixed value of $14 \mathrm{~mm}$ $\mathrm{Hg}$ to predict pulmonary artery pressure ${ }^{30}$. Therefore, we think that the use of a fixed value of 10 
$211 \mathrm{mmHg}$ has little effect on the value of pulmonary artery systolic pressure. All of the above

212 factors increase the heterogeneity of the studies. In our study, the heterogeneity was high, but the

213 generality of this conclusion may be affected by the absence of grouping basis for a subgroup

214 analysis and the inability to conduct further related subgroup analysis. Consequently, our

215 conclusions need to be interpreted with caution.

\section{Conclusions}

217 In summary, echocardiography is a highly sensitive tool for noninvasive screening of POPH.

218 However, if the echocardiography results are abnormal, RHC should be performed to confirm

219 the diagnosis. Our study provides a basis for echocardiography as a POPH screening tool.

220 Moreover, further larger prospective studies are recommended to verify the comprehensive

221 effectiveness of echocardiography as a noninvasive means for detecting patients with POPH.

\section{Acknowledgements}

223 Thanks for all medical workers in hepatology department!

224 Patient consent: Not required.

225 Data sharing statement: No additional data are available.

226

227

228

229

230

231

\section{References}

1. Budhiraja R, Hassoun PM. 2003. Portopulmonary hypertension: a tale of two circulations. Chest 123:562-576 DOI: 10.1378/chest.123.2.562

2. Chen HS, Xing SR, Xu WG, Yang F, Qi XL, Wang LM, Yang CQ. 2013. Porto-pulmonary hypertension in cirrhotic patients: prevalence, clinical features and risk factors. Experimental and Therapeutic Medicine 5:819-824 
232 3. Hua R, Sun YW, Wu ZY, Cheng W, Xu Q, Cao H, Luo M. 2009. Role of 2-dimensional

233 Doppler echocardiography in screening portopulmonary hypertension in portal hypertension

234 patients. Hepatobiliary and Pancreatic Diseases International 8(2):157-161

235

236

237

238

239

240

241

242

243

244

245

246

247

248

249

250

251

252

4. Krowka MJ, Fallon MB, Kawut SM, Fuhrmann V, Heimbach JK, Ramsay MA, Sitbon O, Sokol RJ. 2016. International Liver Transplant Society Practice Guidelines: Diagnosis and Management of Hepatopulmonary Syndrome and Portopulmonary Hypertension.

Transplantation 100(7):1440 DOI: 10.1097/TP.0000000000001229

5. Kawut SM, Krowka MJ, Trotter JF, Roberts KE, Benza RL, Badesch DB, Taichman DB, Horn EM, Zacks S, Kaplowitz N, Brown RS Jr, Fallon MB; Pulmonary Vascular Complications of Liver Disease Study Group. 2008. Clinical risk factors for portopulmonary hypertension. Hepatology 48(1): 196-203 DOI: 10.1002/hep.22275.

6. Le Pavec J, Souza R, Herve P, Lebrec D, Savale L, Tcherakian C, Jais X, YaIci A, Humbert M, Simonneau G, Sitbon O. 2008. Portopulmonary hypertension: survival and prognostic factors. American Journal of Respiratory and Critical Care Medicine 178: 637-643 DOI: 10.1164/rccm.200804-613OC

7. Krowka MJ, Mandell MS, Ramsay MA, Kawut SM, Fallon MB, Manzarbeitia C, Pardo MJ, Marotta P, Uemoto S, Stoffel MP, Benson JT. 2004. Hepatopulmonary syndrome and portopulmonary hypertension: a report of the multicenter liver transplant database. Liver Transplantation 10:174-182 DOI: 10.1002/lt.20016

8. Hadengue A, Benhayoun MK, Lebrec D, Benhamou JP. 1991. Pulmonary hypertension complicating portal hypertension: prevalence and relation to splanchnic hemodynamics. 
9. Martin P, DiMartini A, Feng S, Brown R, Fallon M. 2014. Evaluation for liver transplantation in adults: 2013 practice guideline by the American Association for the Study of Liver Diseases and the American Society of Transplantation. Hepatology 59:1144-1165 DOI: $10.1002 /$ hep. 26972

10. Galie N, Humbert M, Vachiery JL, Gibbs S, Lang I, Torbicki A, Simonneau G, Peacock A, Vonk Noordegraaf A, Beghetti M, Ghofrani A, Gomez Sanchez MA, Hansmann G, Klepetko W, Lancellotti P, Matucci M, McDonagh T, Pierard LA, Trindade PT, Zompatori M, Hoeper M; ESC Scientific Document Group. 2016. 2015 ESC/ERS guidelines for the diagnosis and treatment of pulmonary hypertension: the Joint Task Force for the Diagnosis and Treatment of Pulmonary Hypertension of the European Society of Cardiology (ESC) and the European 19(6): 602-610 DOI: 10.1002/lt.23649

12. Zamora J, Abraira V, Muriel A, Khan KS, Coomarasamy A. 2006. Meta-DiSc: a software for meta-analysis of test accuracy data. BMC Medical Research Methodology 6:31. DOI: 
274 13. Pilatis ND, Jacobs LE, Rerkpattanapipat P, Kotler MN, Owen A, Manzarbeitia C, Reich D,

275 Rothstein K, Munoz SJ. 2000. Clinical predictors of pulmonary hypertension in patients undergoing liver transplant evaluation. Liver Transplantation 6(1): 85-91 DOI: $10.1002 /$ lt.500060116

14. Habash F, Gurram P, Almomani A, Duarte A, Hakeem A, Vallurupalli S, Bhatti S. 2018. Correlation between echocardiographic pulmonary artery pressure estimates and right heart catheterization measurement in liver transplant candidates. Journal of Cardiovascular Imaging 26(2): 75-84 DOI: 10.4250/jcvi.2018.26.e2

15. Colle IO, Moreau R, Godinho E, Belghiti J, Ettori F, Cohen-Solal A, Mal H, Bernuau J, 10.1097/01.tp.0000235520.37189.fe

16. DesJardin JT, Manicardi M, Svetlichnaya Y, Kolaitis NA, Papolos AI, Selby VN, Zier LS, Klein L, Aras MA, Yao FY, Roberts JP, Marco TD. 2019. Noninvasive estimation of pulmonary vascular resistance improves portopulmonary hypertension screening in liver transplant candidates. Clinical Transplantation, 00: e13585 DOI: 10.1111/ctr.13585.

17. Saner FH, Nadalin S, Pavlakovic G, Gu Y, Damink SW, Gensicke J, Fruhauf NR, Paul A, Radtke A, Sotiropoulos GC, Malago M; Broelsch CE. 2006. Portopulmonary hypertension in the early phase following liver transplantation. Transplantation 82(7): 887-891 DOI:

18. Al-Harbi A, Abdullah K, Al-Abdulkareem A, Alghamdi A, Al-Jahdali H. 2014. Prevalence 
295

296

297

298

299

300

301

302

303

304

305

306

307

308

309

310

311

312

313

314

315

of portopulmonary hypertension among liver transplant candidates in a region highly

endemic for viral hepatitis. Annals of Transplantation 19: 1-5 DOI: 10.12659/AOT.889645

19. Torregrosa M, Genesca J, Gonzalez A, Evangelista A, Mora A, Margarit C, Esteban R, Guardia J. 2001. Role of doppler echocardiography in the assessment of portopulmonary hypertension in liver transplantation candidates. Transplantation 71(4): 572-574 DOI: $10.1097 / 00007890-200102270-00015$

20. Cotton CL, Gandhi S, Vaitkus PT, Massad MG, Benedetti E, Mrtek RG, Wiley TE. 2002 Role of echocardiography in detecting portopulmonary hypertension in liver transplant candidates. Liver Transplantation 8(11): 1051-1054 DOI: 10.1053/jlts.2002.35554

21. Devaraj A, Loveridge R, Bosanac D, Stefanidis K, Bernal W, Willars C, Wendon JA, Auzinger J, Desai SR. 2014. Portopulmonary hypertension: improved detection using CT and echocardiography in combination. European Radiology 24(10): 2385-2393 DOI: $10.1007 / \mathrm{s} 00330-014-3289-4$

22. Farzaneh-Far R, McKeown BH, Dang D, Roberts J, Schiller NB, Foster E. 2008. Accuracy of Doppler-estimated pulmonary vascular resistance in patients before liver transplantation. The American Journal of Cardiology 101(2): 259-262 DOI: 10.1016/j.amjcard.2007.07.086

23. Taleb M, Khuder S, Tinkel J, Khouri SJ. 2013. The Diagnostic Accuracy of Doppler Echocardiography in Assessment of Pulmonary Artery Systolic Pressure: A Meta-Analysis. Echocardiography 30(3): 258-265 DOI: 10.1111/echo.12061

24. Krowka MJ, Swanson KL, Frantz RP, McGoon MD, Wiesner RH. 2006. Portopulmonary hypertension: results from a 10-year screening algorithm. Hepatology 44(6): 1502-1510 DOI: 
25. Murray KF, Carithers RL Jr; AASLD. 2005. AASLD practice guidelines: Evaluation of the patient for liver transplantation. Hepatology 41:1407-1432 DOI: 10.1002/hep.20704

26. Janda S, Shahidi N, Gin K, Swiston J. 2011. Diagnostic accuracy of echocardiography for pulmonary hypertension: a systematic review and meta-analysis. Heart 97(8): 612-622 DOI: 10.1136/hrt.2010.212084

27. Posteraro A, Salustri A, Trambaiolo P, Amici E, Gambelli G. 2006. Echocardiographic estimation of pulmonary pressures. Journal of Cardiovascular Medicine 7:545-554 DOI: 10.2459/01.JCM.0000234773.81077.68

28. Garg A, Armstrong WF. 2013. Echocardiography in liver transplant candidates. JACC Cardiovasc Imaging 6:105-119 DOI: 10.1016/j.jcmg.2012.11.002

29. Simonneau G, Montani D, Celermajer DS, Denton CP, Gatzoulis MA, Krowka M, Williams 10.1161/01.cir.70.4.657 
337 Figure 1. Flow diagram of included studies.

338 Figure 2. Forest plot of the combined sensitivity and specificity of echocardiography for screening for POPH in liver transplant candidates.

340 Figure 3 . The summary receiver characteristic curve of the 11 included studies.

341 Figure 4. Forest plot of the correlation between echocardiography and RHC.

342 Figure 5. Deeks' funnel plot for evaluating publication bias. 


\section{Table 1 (on next page)}

Characteristics of studies included in meta-analysis

$\mathrm{POPH}$, portopulmonary hypertension; $\mathrm{FN}$, false negative; $\mathrm{FP}$, false positive; $\mathrm{TN}$, true negative;

$\mathrm{TP}$, true positive. Except for the last one is median age, others are mean age. 
Table 1 Characteristics of studies included in meta-analysis.

\begin{tabular}{|c|c|c|c|c|c|c|c|c|c|}
\hline First author & Year & Sample size & $\begin{array}{c}\text { Mean } \\
\text { /Median age }\end{array}$ & $\begin{array}{c}\text { POPH/Non- } \\
\text { POPH }\end{array}$ & Cut-off value & $\mathbf{T P}$ & $\mathbf{F P}$ & FN & $\mathbf{T N}$ \\
\hline Pilatis ND & 2000 & 55 & 48 & $8 / 47$ & $\mathrm{PASP}>40 \mathrm{mmHg}$ & 5 & 1 & 3 & 46 \\
\hline Raevens S & 2013 & 152 & $58 \pm 11$ & $7 / 145$ & $\mathrm{PASP}>38 \mathrm{mmHg}$ & 7 & 26 & 0 & 119 \\
\hline Habash F & 2018 & 31 & $57 \pm 11$ & $17 / 14$ & PASP $>47 \mathrm{mmHg}$ & 10 & 3 & 7 & 11 \\
\hline Colle IO & 2003 & 165 & $48 \pm 8$ & $10 / 155$ & $\mathrm{PASP}>30 \mathrm{mmHg}$ & 10 & 7 & 0 & 148 \\
\hline DesJardin JT & 2019 & 97 & $56.8 \pm 8.8$ & $11 / 86$ & $\mathrm{PASP} \geq 40 \mathrm{mmHg}$ & 10 & 45 & 1 & 41 \\
\hline Saner FH & 2006 & 74 & $49.6 \pm 11.6$ & $14 / 60$ & $\mathrm{PASP}>40 \mathrm{mmHg}$ & 9 & 14 & 5 & 46 \\
\hline AlHarbi A & 2014 & 248 & $49 \pm 13.9$ & $4 / 244$ & $\mathrm{PASP} \geq 40 \mathrm{mmHg}$ & 4 & 57 & 0 & 187 \\
\hline Hua R & 2009 & 105 & $49.5 \pm 11.8$ & $4 / 101$ & PASP $>30 \mathrm{mmHg}$ & 4 & 18 & 0 & 83 \\
\hline Torregrosa M & 2001 & 107 & $57 \pm 8$ & $5 / 102$ & $\mathrm{PASP} \geq 40 \mathrm{mmHg}$ & 4 & 9 & 1 & 93 \\
\hline Cotton CL & 2002 & 78 & $51 \pm 9.6$ & $11 / 67$ & $\mathrm{PASP} \geq 50 \mathrm{mmHg}$ & 6 & 10 & 5 & 57 \\
\hline Devaraj A & 2014 & 48 & 54 & $5 / 43$ & $\mathrm{PASP} \geq 40 \mathrm{mmHg}$ & 5 & 18 & 0 & 25 \\
\hline
\end{tabular}

2 Notes: POPH, portopulmonary hypertension; FN, false negative; FP, false positive; TN, true negative; TP, true positive. Except for the

3 last one is median age, others are mean age. 
Figure 1

Flow diagram of included studies.
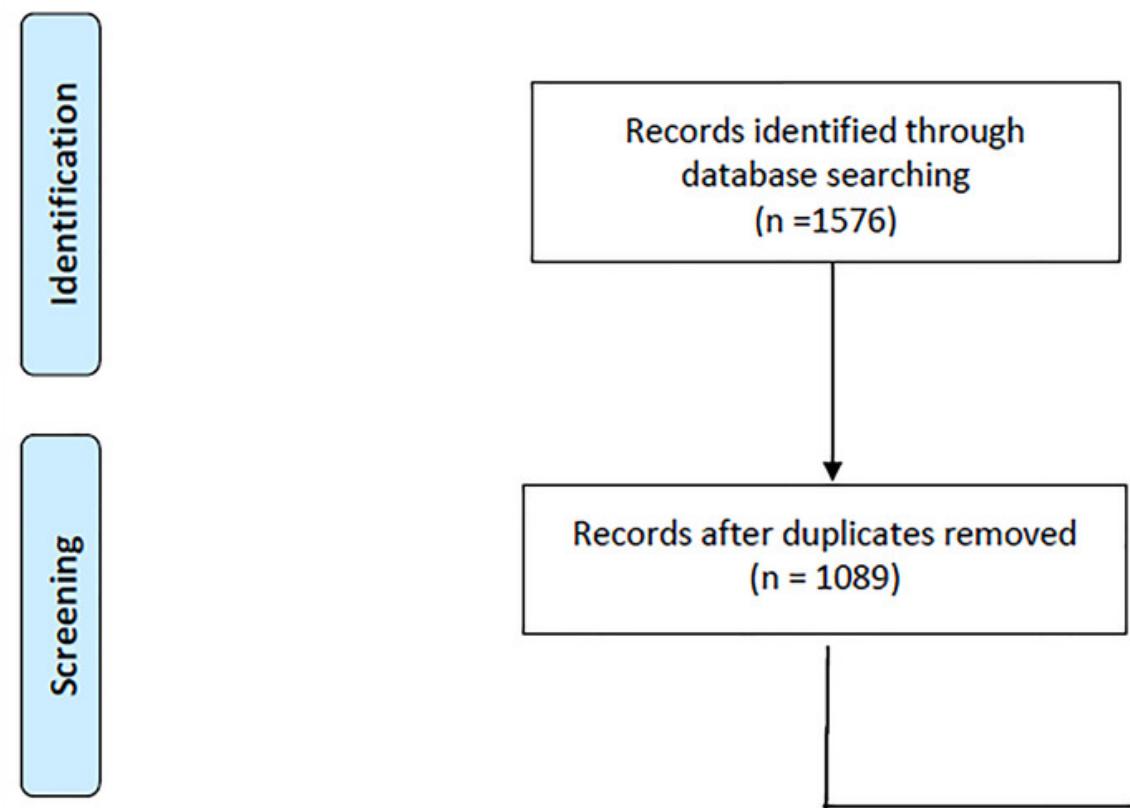

Records after duplicates removed ( $n=1089$ )
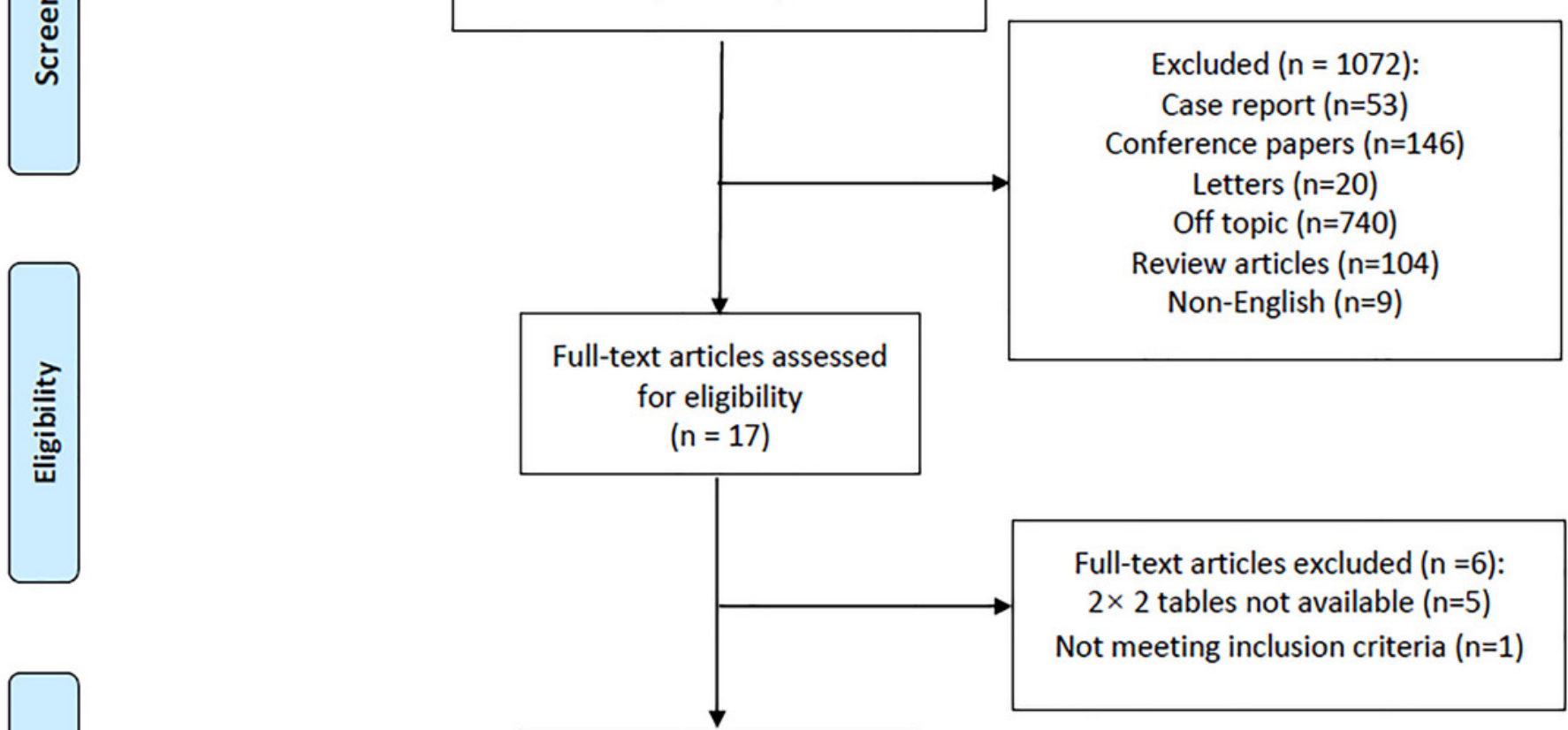

\section{륨}

Studies included in quantitative synthesis (meta-analysis)

$$
(n=11)
$$


Figure 2

Forest plot of the combined sensitivity and specificity of echocardiography for screening for POPH in liver transplant candidates.
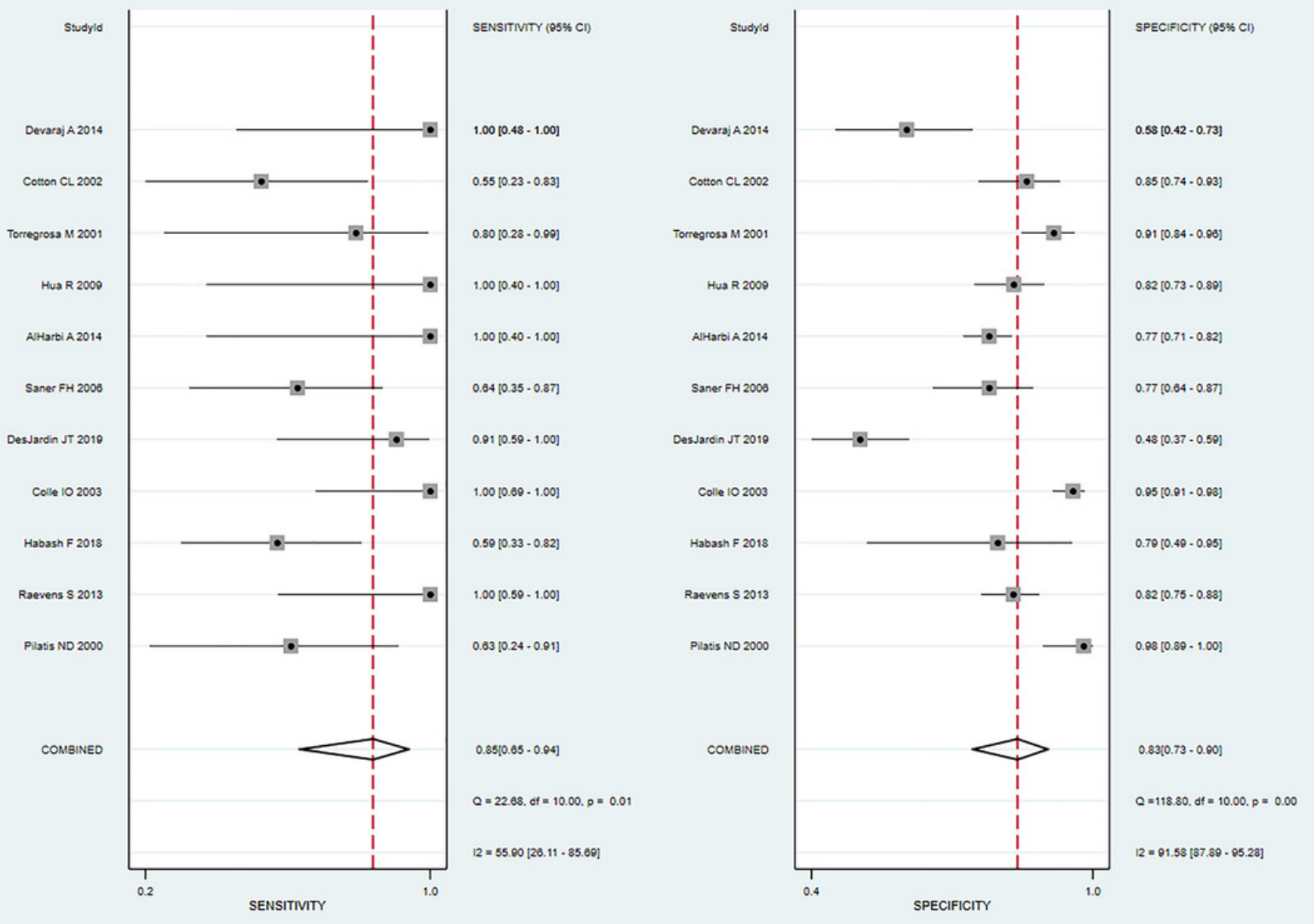
Figure 3

The summary receiver characteristic curve of the 11 included studies.

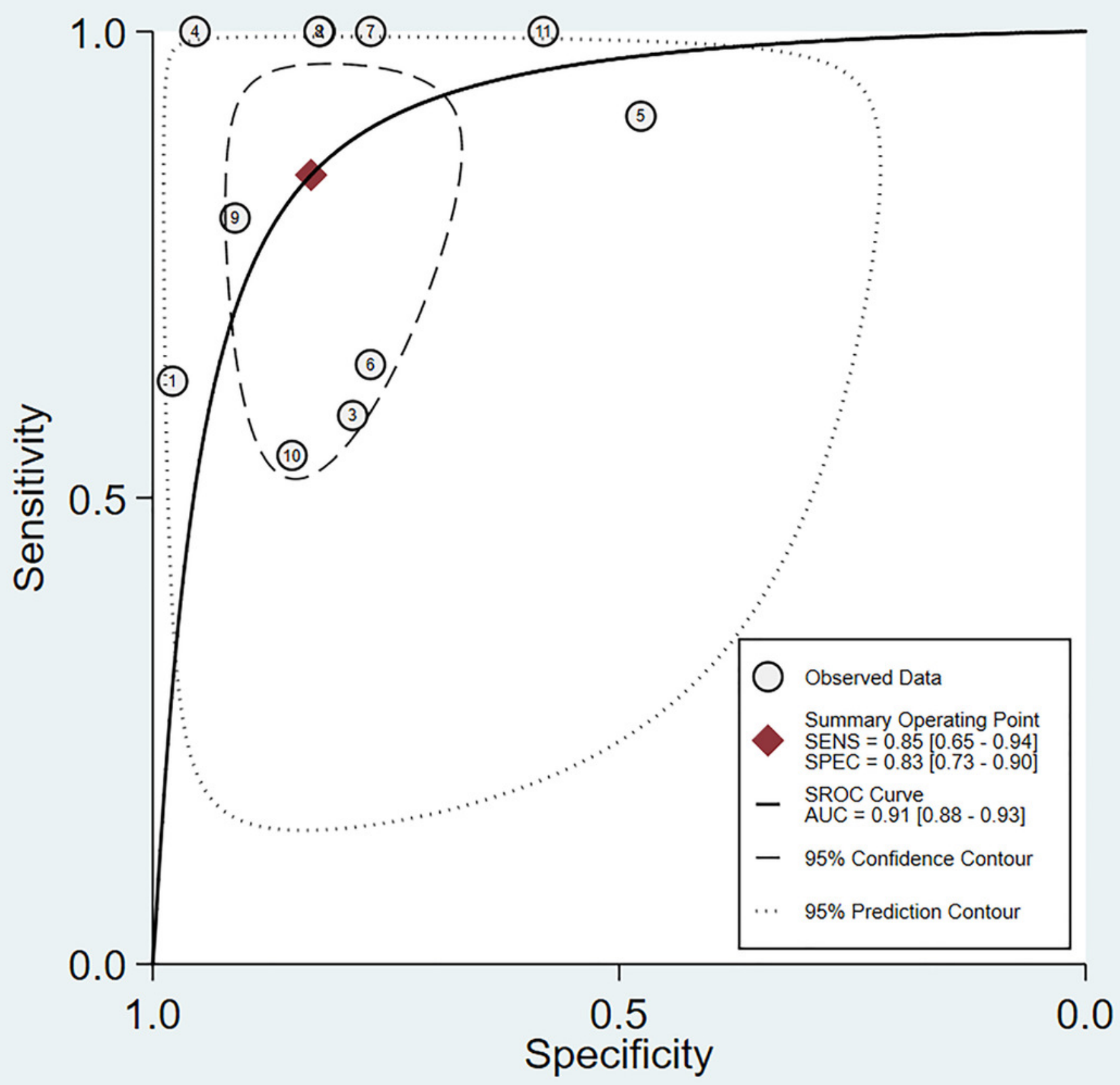


Figure 4

Forest plot of the correlation between echocardiography and RHC.

Study

Farzaneh-Far R et al. 2008

Cotton CL et al. 2002

Kim WR et al. 2000

Random effects model

Heterogeneity: $\mathrm{I}^{2}=86 \%, \mathrm{t}^{2}=0.1266, \mathrm{p}<0.0$
Total

22

78

74

174
Correlation

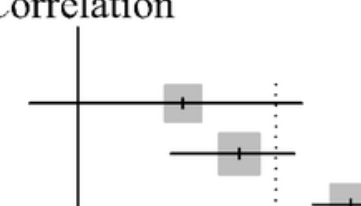

COR

$0.30 \quad[-0.14 ; 0.64] \quad 28.1 \%$

$0.46 \quad[0.26 ; 0.62] \quad 36.0 \%$

$0.78 \quad[0.67 ; 0.86] \quad 35.8 \%$

$0.57[0.20 ; 0.79] \quad 100.0 \%$

$95 \%-$ CI Weight

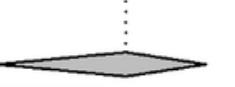

0.5 
Figure 5

Deeks' funnel plot for evaluating publication bias.

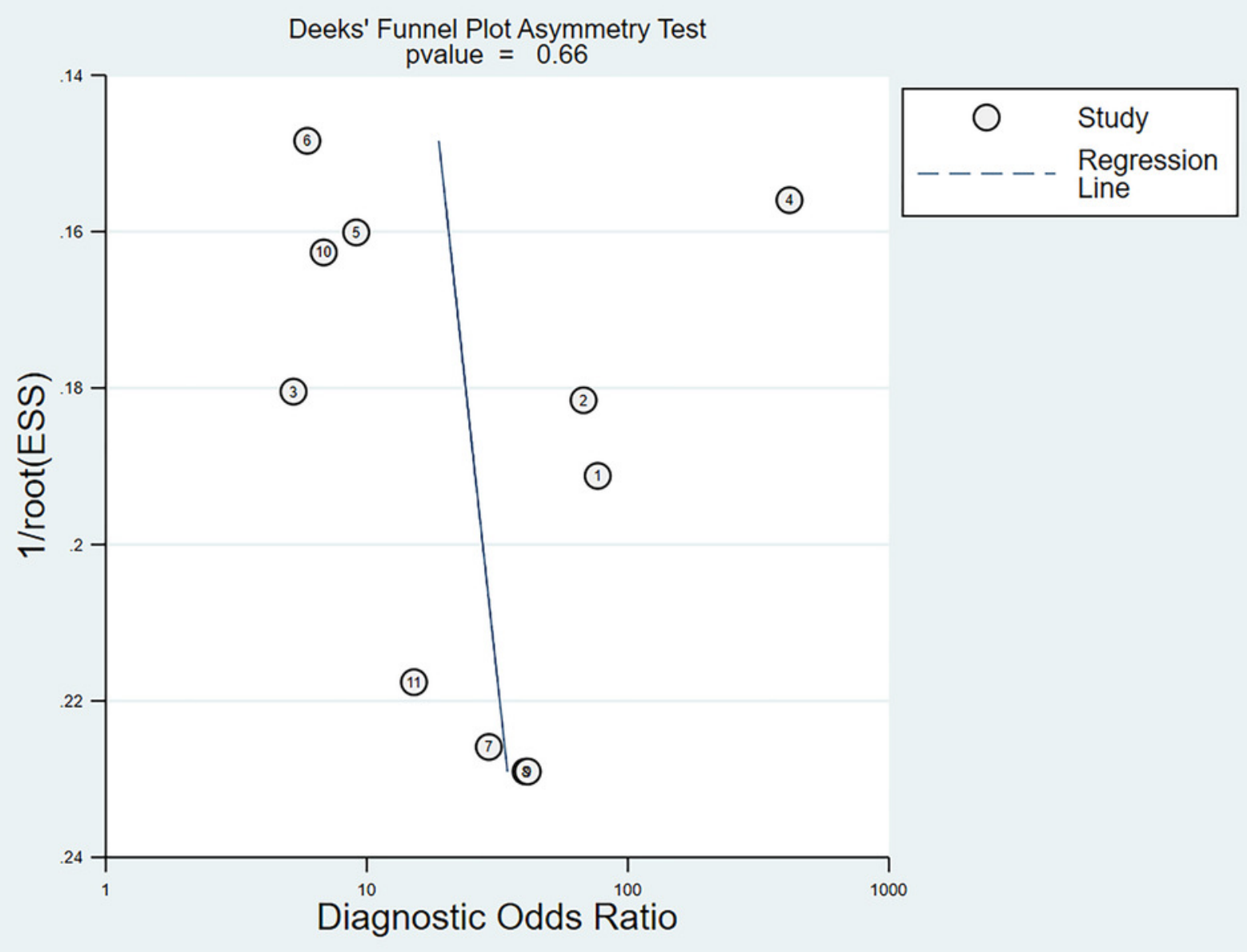

